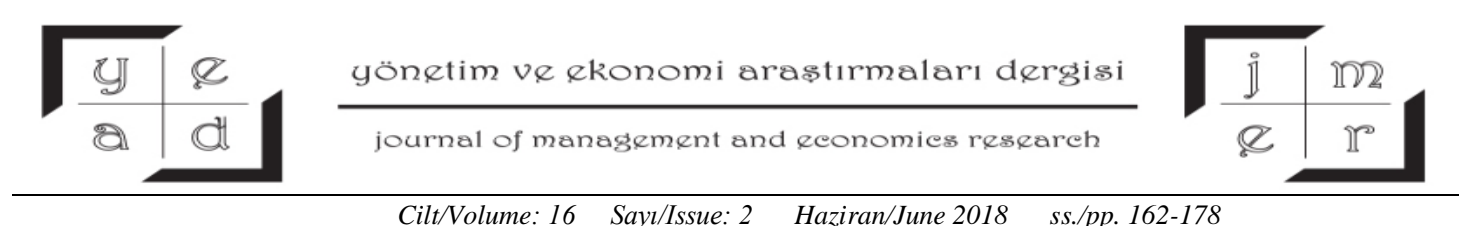

Cilt/Volume: $16 \quad$ Sayl/Issue: 2 Haziran/June 2018 ss./pp. 162-178

M.T. Bilişik Doi: http://dx.doi.org/10.11611/yead.398967

\title{
FAILURE MODE AND EFFECT ANALYSIS AND IMPLEMENTATION IN A TEXTILE FACTORY
}

\section{Asst. Prof. Murat Taha BíLişíiK * iD}

\begin{abstract}
Quality concept has been keeping on its importance by assimilating and developing the customer service orientation. The companies which satisfy customer requests properly and reasonably get more powerful relationship compared with competitors. So, the companies have to provide the best services that customers deserve. With that study which performed in a textile plant, risk priority numbers before and after the improvement is calculated for faults met and for faults which may appear later. To apply Failure Mode and Affect Analysis (FMEA), firstly, a decision team has been established to determine the causes of faults. And then FMEA has been performed to prioritize the critical potential failure modes of the process. Finally, after improvement, some recommended actions regarding the findings were discussed.
\end{abstract}

Keywords: Failure Mode and Effect Analysis, Risk Priority Number, Textile

JEL Codes: L15, L67, O32

\section{HATA TÜRÜ VE ETKILERİ ANALİZİ VE TEKSTILL SEKTÖRÜNDE BİR UYGULAMA}

\section{ÖZ}

Kalite kavramı müşteri hizmetini özümsemeyi ve odaklanmayı dayalı olarak önemini sürdürmektedir. Müşteri ihtiyaçlarını tutarlı ve makul şekilde karşılayabilen işletmeler, rakiplerine göre müssterileriyle daha güçlü ilişkiler gelişstirmektedir. Bu nedenle, işletmeler müşterilerin hak ettiği en iyi hizmeti sunmalıdır. Bir tekstil fabrikasında gerçekleştirilen bu çalışmada, yapılmış olan bir iyileştirme çalışmasından önceki ve iyileştirme çalışmasından sonraki saptanan ve karşılaşabilecek hataları ölçen risk öncelik sayıları hesaplanmıştır. Hata türü ve etkileri analizi uygulamasında, hata nedenlerini araştırmak üzere bir karar takımı oluşturulmuştur. Ardından, hata türü ve etkileri analizi uygulanmış olup süreçte meydana gelen kritik potansiyel hata türleri önceliklendirilmiştir. Son olarak, iyileştirme ardından, bulgulara ilişkin öneriler tartışılmıştır..

Anahtar Kelimeler: Hata Türü ve Etkileri Analizi, Risk Öncelik Sayısl, Tekstil.

\footnotetext{
* İstanbul Kültür Üniversitesi, m.bilisik@iku.edu.tr
} 
JEL Siniflandirmast: L15, L67, O32

\section{1. INTRODUCTION}

Quality concept imprtance is getting appreciated day by day which become compulsory. As the customers identify the quality issue, the customer satisfaction should be maximized. So, to accomplish the objective, we have to provide service faultlessly and reliably.

FMEA determines the risks which cause failure, also array the reason and effects of these failures by proper improvement techniques. Moreover, in order to minimize failures and prevent faulty items on the further processes, related optimization techniques may be used.

The goal of any FMEA study is to identify what might go wrong before an error is actually made, whether that is an error in design or in realization of the design. Thus, a systematic approach is used to assess these potential errors in order to quantitatively prioritize risk (Anleitner,2010). FMEA, requirements on quality assurance, techniques for solving problems, chairmanship of meetings, and auditor training(Müller, 1995). Whereas, failure modes and effects analysis (FMEA) is amethod utilized in order to determine and classify failure types with respect to product development, service, system and improvement of the processes (Eleren, 2007).

Failure mode and effect analysis (FMEA) is an important method for designing and prioritising preventive maintenance activities and is often used as the basis for preventive maintenance planning. (Braaksma et al. 1055-1071)

Preventing process and product problems before they occur is the purpose of Failure Mode and Effect Analysis (FMEA). Used in both the design and manufacturing processes, they substantially reduce costs by identifying product and process improvements early in the develop process when changes are relatively easy and inexpensive to make. The result is a more robust process because the need for after-the-fact corrective action and late change crises are reduced or eliminated (Raymond 2008).

There are many studies to be found in the literature regarding FMEA. FMEA flight control system came into use flight control system development in USA at teh beginning of 1950 which kept using on aerospace industry regularly after 1960. In case of civillian use, Ford developed for automative industry firstly and after 1972, FMEA worked out for Ford. (Akın, 1998:12).

In 1993, Automative Industry Action Group (AIAG) and American Society for Quality Control (ASQC) created FMEA standardization for industry. That standart FMEA were accepted by Chrysler, Ford ve General Motors which made a corparation for developing of QS 9000. Recently, many industries carry on formal FMEA standards and also FMEA, QS 9000, ISO / TS 16949, ISO 9001:2000 and other quality management systems have become a necessity today. Upon reviewing 
literature, FMEA technique can be used in several studies carried out within the scope of different disciplines until today. Today it is also used in different industries widely.

The goals of FMEA are as follows: (Ozyazgan, 2014)

- Defining potential error / fault types, rates, effects and the degree of importance

- Identify the critical and determinant characteristics

- Sorting potential errors of design and process based on severity

- Identifying and testing to eliminate or minimize errors, defects, malfunctions, and changes and to ensure product development

- Avoid potential errors that may occur along to the product or process, by predefining them

- Eliminate potential types of errors to take corrective actions or reduce the posibility of formation

The benefits of FMEA are as follows: (Y1lmaz, 2000);

- Assisting to error reduce and investigating faults in this way,

- Increasing of customer satisfaction

- It is describe to missing points of production safety, production technology safety

- Cost reduced by preventing mistakes.

- Decreasing of marketing time.

FMEA needs detailed information for each phase/component of the system, thus making possible to analyze the modalities in which each phase/component can fail. The required information is not always readily available.

Types of Failure Mode and Effect Analysis;

FMEA method is basically handled in four ways; (Raymond, 2008)

Product FMEA: Product FMEAs can be conducted at each phase in the design process (preliminary design, prototype, or final design), or they can be used on products that are already in production.

Design FMEA: The objective for a design FMEA is to uncover problems with the product that will result in safety hazards, product malfunctions, or a shortened product life. The key question asked in design FMEAs is: How can the product fail?

Process FMEA: Process FMEAs uncover process problems related to the manufacture of the product. It is helpful when conducting a process FMEA to think in terms of the five elements of a process: people, materials, equipment, methods, and environment. 
Service FMEA: It is a method to analyze the service before it reaches to the customer.

\section{APPLICATION STEPS OF FAILURE MODE AND EFFECT ANALYSIS}

A basic FMEA application contains calculation of defect types, reasons, probabilities, severity and discoverability and risk priority number of the functions respectively and listing them from higher to smaller and taking some measures to decrease risk.

Implementation phases of FMEA technique are as follows:

- Setting of FMEA team and detecting process or processes to be analyzed as below list;

- Identifying failure modes

- Identifying potential effect or effects of the failure

- Identifying causes of failures

- Identifying failure severity

- Identifying failure cccurrence

- Identifying detectability condition of failures

- Calculating Risk Priority Numbers (RPN)

- Making proposals thereby paying attention to values calculated

- Carrying out regulatory or preventive applications

- Comparing RPN numbers with priorities after improvement

The risk of possible failures is assessed using the risk priority number (RPN), which is calculated on the basis of assessment of failure severity, probability of occurrence and probability of detection. (Vykydal, 2015)

\section{RPN $=$ Occurrence $\times$ Severity $\times$ Detection}

This is the way the product of these three values RPN (Risk Priority Number) is obtained (Roszak et al. 449-451).

Severity is the value of item by customer side after error occured (Yak1t, 2011). The second criterion involves potential technical causes used to estimate the probability of impact risk occurrence (O). Finally, one can estimate the possibility of influence of the causes and the related risk. For the criteria used to evaluate the importance of environmental impact (S), the probability of cause occurrence $(\mathrm{O})$ and for the causes of influence (D), like in the quality area, values in the range of 1 (small risk) to 10 (high risk) are assigned.

In case of occurrence, 1 is remote probability of occurrence. If the prosess under control seen as low probability of occurrence as statistically, it is numbered between 2-5. If the problem is moderate, 
but process is under control, this is 6 . If the problem frequency is high, this is numbered as 7-8. In case of very high probability of occurrence, it is 9-10.(Sabir and Bebekli,2015)

According to the Ford Motor company ranges of values specified for the decision-making: (Bertsche, 2008)

- $\mathrm{RPN}<40$ is no need to take measures

- $40 \leq \mathrm{RPN} \leq 100$ is worth taking the precaution

- RPN $>100$ should take precautions if necessary

If two or more errors have the same value of RPN, firstly with high intensity and then with high deviation ones should be addressed

After theresults have been recorded, it is time to confirm, evaluate, and measure the success or failure.

This evaluation takes the form of three basic questions (Stamatis, 2003)

- Is the situation better than before?

- Is the situation worse than before?

- Is the situation the same as before?

Number of RPN has no value or meaning. Just enable you to sort and compared errors to each other in terms of criticality and show the relative importance. It gives a general idea of the envisaged system. Corrective action will begin according to the RPN depending on the value of the error causes which are analyzed by lining up critical points and with the highest cause of the error. (Ozyazgan, 2014)

Success of FMEA belongs to make right availeble and potential error determination, and action plan to this errors. So FMEA have to accepted by all company workers.

\section{IMPLEMENTATION}

Implementation technique phases of FMEA indicated are performed in a textile company for each part of factor which are yarn production, weaving and dyehouse in a row during 10 month. First of all, FMEA team was formed from production managers, production engineers, maintenence chiefs. This team has determined the potential failures, effects, and causes of the failures trough brainstorming and using cause-and effect diagrams. When we score the rating of severity, occurrence, detection to reach RPN value, we consulted to customer feedbacks, quality control sheets, our observations and experience of FMEA team. Determined all datas were recorded and created RPN table. The causes with higher RPNs were considered as the more important causes, which would be selected and solved further. 


\subsection{Yarn Production Part}

In this study, we described 6 available and potential error with FMEA team in yarn production part.

Figure 1. Error Types For Yarn Production Failures

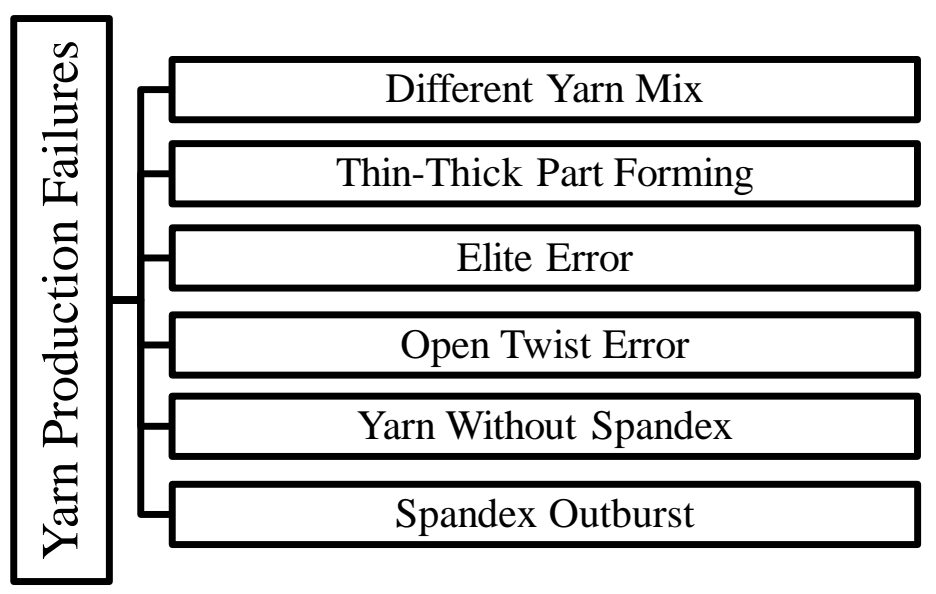

Regarding described error in yarn production part, as firts step, we determined the effects of the failures and causes of failures with FMEA Team. Hereby, we reached First RPN Value.

After our actions with FMEA team to errors, we got Final RPV Value for each fault which showed below Table1.

Table 1. Yarn Production Part Fmea Application

\begin{tabular}{|c|c|c|c|c|c|c|c|c|}
\hline & $\begin{array}{c}\text { Function } \\
\text { Mode }\end{array}$ & $\begin{array}{l}\text { Failure } \\
\text { Modes }\end{array}$ & $\begin{array}{l}\text { Effects of the } \\
\text { failure }\end{array}$ & $\begin{array}{l}\text { Causes of } \\
\text { Failures }\end{array}$ & $\begin{array}{l}\text { First } \\
\text { RPN } \\
\text { Valu } \\
\text { e }\end{array}$ & $\begin{array}{c}\text { Recommended } \\
\text { Activity }\end{array}$ & $\begin{array}{c}\text { Undertaken } \\
\text { Activity }\end{array}$ & $\begin{array}{c}\text { Final } \\
\text { RPN } \\
\text { Valu } \\
\text { e }\end{array}$ \\
\hline 1 & $\begin{array}{c}\text { Yarn } \\
\text { Production }\end{array}$ & $\begin{array}{l}\text { Different } \\
\text { yarn mix }\end{array}$ & $\begin{array}{l}\text { faulty fabric } \\
\text { production by } \\
\text { using different } \\
\text { yarn bobbin }\end{array}$ & $\begin{array}{l}\text { Stocking of same } \\
\text { color-different } \\
\text { bobbin together in } \\
\text { same place }\end{array}$ & 160 & $\begin{array}{l}\text { Stocking of } \\
\text { different yarn } \\
\text { bobbin on } \\
\text { different places }\end{array}$ & $\begin{array}{l}\text { Different yarn } \\
\text { bobbins are } \\
\text { stocked } \\
\text { different } \\
\text { places and all } \\
\text { lines are } \\
\text { marked }\end{array}$ & 120 \\
\hline
\end{tabular}


Yönetim ve Ekonomi Arasttrmaları Dergisi / Journal of Management and Economics Research

Cilt/Volume: $16 \quad$ Sayl/Issue: 2 Haziran/June 2018 ss./pp. 162-178 M.T. Bilişik Doi: http://dx.doi.org/10.11611/yead.398967

\begin{tabular}{|c|c|c|c|c|c|c|c|c|}
\hline 2 & $\begin{array}{c}\text { Yarn } \\
\text { Production }\end{array}$ & $\begin{array}{l}\text { Thin- } \\
\text { Thick } \\
\text { Yarn } \\
\text { Forming }\end{array}$ & $\begin{array}{c}\text { Not able to } \\
\text { produce } \\
\text { homogen } \\
\text { thickness yarn on } \\
\text { production which } \\
\text { will effect quality } \\
\text { of fabric }\end{array}$ & $\begin{array}{l}\text { Dirtness of some } \\
\text { aparatus related } \\
\text { with yarn } \\
\text { machine or defect } \\
\text { of machine } \\
\text { aparatus }\end{array}$ & 120 & $\begin{array}{l}\text { Increasing of } \\
\text { peer-reviewed } \\
\text { control of } \\
\text { related aparatus } \\
\text { and bobbin }\end{array}$ & $\begin{array}{l}\text { No of peer- } \\
\text { reviewed } \\
\text { control of } \\
\text { related } \\
\text { machine } \\
\text { aparatus and } \\
\text { bobbin control } \\
\text { is increased }\end{array}$ & 72 \\
\hline 3 & $\begin{array}{c}\text { Yarn } \\
\text { Production }\end{array}$ & $\begin{array}{l}\text { Elite } \\
\text { Error }\end{array}$ & $\begin{array}{l}\text { Occuring of Neps } \\
\text { unevenness on the } \\
\text { yarn }\end{array}$ & $\begin{array}{c}\text { Mechanical } \\
\text { instability of } \\
\text { twisting machine } \\
\text { aparatus cause } \\
\text { this }\end{array}$ & 84 & $\begin{array}{l}\text { İncreasing of } \\
\text { twisting } \\
\text { aparatus } \\
\text { changing peirod }\end{array}$ & $\begin{array}{c}\text { twisting } \\
\text { aparatus } \\
\text { changing } \\
\text { peirod have } \\
\text { been increased }\end{array}$ & 63 \\
\hline 4 & $\begin{array}{c}\text { Yarn } \\
\text { Production }\end{array}$ & $\begin{array}{l}\text { Open } \\
\text { Twist } \\
\text { Error }\end{array}$ & $\begin{array}{c}\text { Not to reach } \\
\text { required twist } \\
\text { and getting loose } \\
\text { yarn which } \\
\text { effect quality of } \\
\text { fabric }\end{array}$ & $\begin{array}{c}\text { Abnormal } \\
\text { working condions } \\
\text { of belt system on } \\
\text { ring machine } \\
\text { cause this } \\
\text { problem }\end{array}$ & 128 & $\begin{array}{c}\text { Controlling } \\
\text { period of belt } \\
\text { system on ring } \\
\text { machine which } \\
\text { provide } \\
\text { movement } \\
\text { makes as } \\
\text { weekly }\end{array}$ & $\begin{array}{l}\text { Belt system } \\
\text { control has } \\
\text { been done as } \\
\text { weekly }\end{array}$ & 96 \\
\hline 5 & $\begin{array}{c}\text { Yarn } \\
\text { Production }\end{array}$ & $\begin{array}{c}\text { Yarn } \\
\text { Without } \\
\text { Spandex }\end{array}$ & $\begin{array}{c}\text { Not to get target } \\
\text { yarn by breaking } \\
\text { of spandex on } \\
\text { connecting of } \\
\text { spandex and rigid } \\
\text { yarn }\end{array}$ & $\begin{array}{l}\text { Damaging of } \\
\text { spandex shift } \\
\text { other carriers on } \\
\text { machine which } \\
\text { connnect } \\
\text { spandex and rigid } \\
\text { yarn break the } \\
\text { spandex }\end{array}$ & 90 & $\begin{array}{c}\text { Controling of } \\
\text { spandex shift by } \\
\text { operators } \\
\text { periodically }\end{array}$ & $\begin{array}{l}\text { Controling of } \\
\text { spandex shift } \\
\text { by operators } \\
\text { has been done } \\
\text { as daily }\end{array}$ & 72 \\
\hline 6 & $\begin{array}{c}\text { Yarn } \\
\text { Production }\end{array}$ & $\begin{array}{l}\text { Spandex } \\
\text { Outburst }\end{array}$ & $\begin{array}{c}\text { visibility of } \\
\text { spandex after } \\
\text { fabric production } \\
\text { because of } \\
\text { uncentering } \\
\text { spandex on yarn } \\
\text { production }\end{array}$ & $\begin{array}{c}\text { Imperfection } \\
\text { work of aparatus } \\
\text { which makes the } \\
\text { spandex centered } \\
\text { in ridig item on } \\
\text { yarn machine }\end{array}$ & 72 & $\begin{array}{c}\text { Increasing of } \\
\text { control of } \\
\text { aparatus which } \\
\text { makes the } \\
\text { spandex } \\
\text { centered by } \\
\text { operators }\end{array}$ & $\begin{array}{l}\text { Controling of } \\
\text { related } \\
\text { aparatus is } \\
\text { provided } \\
\text { regularly by } \\
\text { visual control }\end{array}$ & 60 \\
\hline
\end{tabular}


As we mentioned before, RPN can be calculated by multiplying S (severity), O (occurrence) and D (detectability) for each type of error.

At below table, fault possibilities, density values and detectability values of FMEA study for the relevant product were calculated.

Table 2. Yarn Production First And Final Rpn Comparison

\begin{tabular}{|c|c|c|c|c|c|c|c|c|c|}
\hline $\begin{array}{c}\text { Functio } \\
\text { n Mode } \\
\text { Line }\end{array}$ & $\begin{array}{c}\text { Severit } \\
\mathbf{y}\end{array}$ & $\begin{array}{c}\text { Occurrenc } \\
\text { e }\end{array}$ & $\begin{array}{c}\text { Detectio } \\
\text { n }\end{array}$ & $\begin{array}{c}\text { First } \\
\text { RPN } \\
\text { Valu } \\
\text { e }\end{array}$ & $\begin{array}{c}\text { Severit } \\
\mathbf{y}\end{array}$ & $\begin{array}{c}\text { Occurrenc } \\
\text { e }\end{array}$ & $\begin{array}{c}\text { Detectio } \\
\text { n }\end{array}$ & $\begin{array}{c}\text { Final } \\
\text { RPN } \\
\text { Valu } \\
\text { e }\end{array}$ & $\begin{array}{c}\text { RPN } \\
\% \\
\text { Chang } \\
\text { e }\end{array}$ \\
\hline 1 & 8 & 4 & 5 & 160 & 8 & 3 & 5 & 120 & 25 \\
\hline 2 & 6 & 5 & 4 & 120 & 6 & 3 & 4 & 72 & 40 \\
\hline 3 & 7 & 4 & 3 & 84 & 7 & 3 & 3 & 63 & 25 \\
\hline 4 & 8 & 4 & 4 & 128 & 8 & 3 & 4 & 96 & 25 \\
\hline 5 & 6 & 5 & 3 & 90 & 6 & 4 & 3 & 72 & 20 \\
\hline 6 & 6 & 6 & 2 & 72 & 6 & 5 & 2 & 60 & 16 \\
\hline
\end{tabular}

As we investigate causes of faults and undertaken activities, abnormal machine aparatus condition or insufficient control by operators are observed as source of main reason of errors in yarn production part. Periodic control of relevant aparatus and operators education was increased. With this study we get $25 \%$ quality progress in yarn production part.

\subsection{Weaving Part}

In weaving part, there are some preparation process before weaving which increased possibility of creating error. We have got 10 available and potential errors with FMEA team by using brainstorming. 
Figure 2. Error Types For Weaving Failures

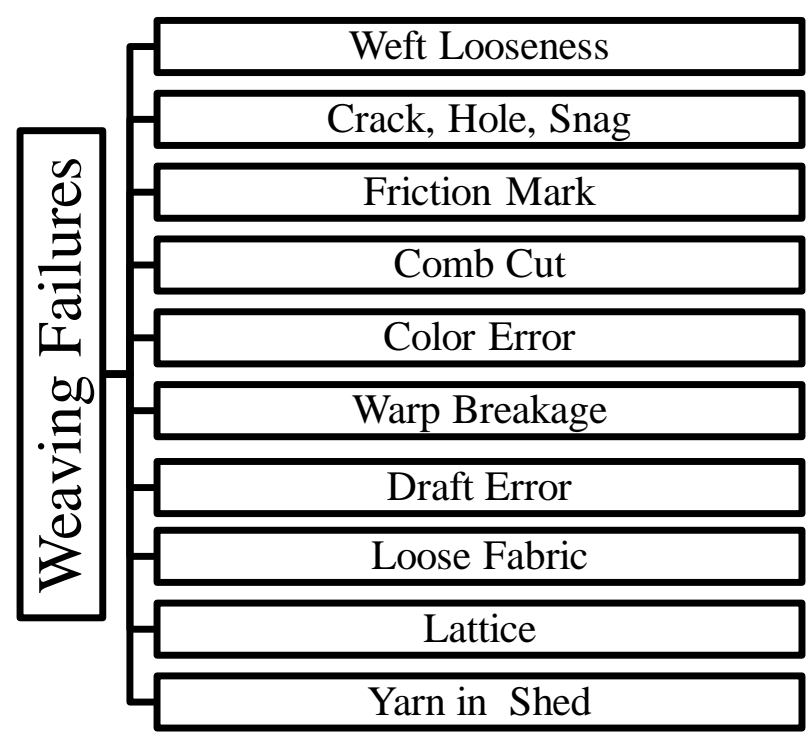

Based on described error in yarn production part, as firts step we determined effects of the failures and causes of failures with FMEA Team. Hereby, we reached First RPN Value.

After our actions with FMEA team to errors, we got Final RPV Value for each fault which showed at below table 3;

Table 3. Weaving Part Fmea Application

\begin{tabular}{|c|c|c|c|c|c|c|c|c|}
\hline & $\begin{array}{c}\text { Function } \\
\text { Mode }\end{array}$ & $\begin{array}{l}\text { Failure } \\
\text { Modes }\end{array}$ & $\begin{array}{l}\text { Effects of the } \\
\text { failure }\end{array}$ & $\begin{array}{l}\text { Causes of } \\
\text { Failures }\end{array}$ & $\begin{array}{l}\text { First } \\
\text { RPN } \\
\text { Valu } \\
\text { e }\end{array}$ & $\begin{array}{c}\text { Recommended } \\
\text { Activity }\end{array}$ & $\begin{array}{c}\text { Undertaken } \\
\text { Activity }\end{array}$ & $\begin{array}{c}\text { Final } \\
\text { RPN } \\
\text { Valu } \\
\text { e }\end{array}$ \\
\hline 1 & $\begin{array}{c}\text { Weaving } \\
\text { Part }\end{array}$ & $\begin{array}{c}\text { Weft } \\
\text { Loosenes } \\
\text { s }\end{array}$ & $\begin{array}{l}\text { Hole during } \\
\text { weft wise } \\
\text { because of } \\
\text { lacking of } \\
\text { weft yarn in } \\
\text { shed }\end{array}$ & $\begin{array}{c}\text { Machine } \\
\text { instability, } \\
\text { abnormal shed } \\
\text { closure degree or } \\
\text { low weft yarn } \\
\text { strength }\end{array}$ & 180 & $\begin{array}{l}\text { Increase the } \\
\text { controling of } \\
\text { related machine } \\
\text { aparatus and weft } \\
\text { yarn strength }\end{array}$ & $\begin{array}{c}\text { weft yarn } \\
\text { strength and the } \\
\text { controling of } \\
\text { related machine } \\
\text { aparatus are } \\
\text { provided }\end{array}$ & 120 \\
\hline 2 & $\begin{array}{c}\text { Weaving } \\
\text { Part }\end{array}$ & $\begin{array}{l}\text { Crack, } \\
\text { Hole, } \\
\text { Snag }\end{array}$ & $\begin{array}{l}\text { Damaged part } \\
\text { formation on } \\
\text { surface of } \\
\text { fabric }\end{array}$ & $\begin{array}{l}\text { Some mechanical } \\
\text { breakdowns, Bad } \\
\text { transfering of } \\
\text { fabric roll, or } \\
\text { foreign body }\end{array}$ & 108 & $\begin{array}{l}\text { To educate the } \\
\text { operators } \\
\text { regarding fabric } \\
\text { roll transfering } \\
\text { and not use sharp }\end{array}$ & $\begin{array}{l}\text { Operators are } \\
\text { educated } \\
\text { regarding fabric } \\
\text { roll transfering } \\
\text { snd using of }\end{array}$ & 72 \\
\hline
\end{tabular}




\begin{tabular}{|c|c|c|c|c|c|c|c|c|}
\hline & & & & damage the fabric & & $\begin{array}{l}\text { and small items } \\
\text { by the machine }\end{array}$ & $\begin{array}{c}\text { sharp and small } \\
\text { items are } \\
\text { banned by the } \\
\text { machine }\end{array}$ & \\
\hline 3 & $\begin{array}{c}\text { Weaving } \\
\text { Part }\end{array}$ & $\begin{array}{c}\text { Friction } \\
\text { mark }\end{array}$ & $\begin{array}{l}\text { Some } \\
\text { continuous } \\
\text { lines like } \\
\text { shining or } \\
\text { tone through } \\
\text { the warp wise } \\
\text { on fabric }\end{array}$ & $\begin{array}{l}\text { Unbalanced } \\
\text { operation of weft } \\
\text { yarn carrier, } \\
\text { inadequate shed } \\
\text { or some burrs on } \\
\text { moving parts }\end{array}$ & 100 & $\begin{array}{c}\text { Making of } \\
\text { mechanical } \\
\text { control of moving } \\
\text { parts and } \\
\text { checking of } \\
\text { surface of } \\
\text { aparatus regularly }\end{array}$ & $\begin{array}{l}\text { Controls of } \\
\text { moving parts } \\
\text { and surface of } \\
\text { item are } \\
\text { provided }\end{array}$ & 75 \\
\hline 4 & $\begin{array}{c}\text { Weaving } \\
\text { Part }\end{array}$ & $\begin{array}{c}\text { Comb } \\
\text { Cut }\end{array}$ & $\begin{array}{l}\text { Cutting of } \\
\text { weft yarn } \\
\text { within shed }\end{array}$ & $\begin{array}{c}\text { Damaged comb, } \\
\text { high warp tension } \\
\text { or high weft } \\
\text { density }\end{array}$ & 112 & $\begin{array}{l}\text { Increase the } \\
\text { controling of } \\
\text { related machine } \\
\text { aparatus and warp } \\
\text { tension control for } \\
\text { evey yarn on } \\
\text { beam }\end{array}$ & $\begin{array}{l}\text { Aparatus control } \\
\text { and warp } \\
\text { tension control } \\
\text { for evey yarn on } \\
\text { beam are done } \\
\text { and }\end{array}$ & 56 \\
\hline 5 & $\begin{array}{c}\text { Weaving } \\
\text { Part }\end{array}$ & $\begin{array}{l}\text { Color } \\
\text { Error }\end{array}$ & $\begin{array}{l}\text { Deviation on } \\
\text { fabric due to } \\
\text { wrong pattern } \\
\text { repeat of warp } \\
\text { yarn }\end{array}$ & $\begin{array}{l}\text { When the } \\
\text { breakage happen } \\
\text { on the machine, } \\
\text { operators tie the } \\
\text { wrong warp yarn } \\
\text { or draft fault }\end{array}$ & 216 & $\begin{array}{l}\text { To provide the } \\
\text { pattern control } \\
\text { after yarn cut and } \\
\text { loading the } \\
\text { machine new yarn } \\
\text { on beam }\end{array}$ & $\begin{array}{c}\text { Operators are } \\
\text { educated } \\
\text { regarding } \\
\text { pattern control. }\end{array}$ & 108 \\
\hline 6 & $\begin{array}{c}\text { Weaving } \\
\text { Part }\end{array}$ & $\begin{array}{c}\text { warp } \\
\text { breakage }\end{array}$ & $\begin{array}{c}\text { Weaving } \\
\text { disorder } \\
\text { becasue of } \\
\text { non fall of } \\
\text { drop wire and } \\
\text { making hole } \\
\text { on fabric } \\
\text { surface }\end{array}$ & $\begin{array}{l}\text { Non fall of drop } \\
\text { wire or non drop } \\
\text { wire on draft } \\
\text { process }\end{array}$ & 147 & $\begin{array}{l}\text { To educate the } \\
\text { operators } \\
\text { regarding } \\
\text { optimum fabric } \\
\text { surface }\end{array}$ & $\begin{array}{l}\text { Operators are } \\
\text { educated to } \\
\text { check fabric } \\
\text { structure ever so } \\
\text { often }\end{array}$ & 105 \\
\hline 7 & $\begin{array}{c}\text { Weaving } \\
\text { Part }\end{array}$ & $\begin{array}{l}\text { Draft } \\
\text { Error }\end{array}$ & $\begin{array}{l}\text { Error on fabric } \\
\text { surface } \\
\text { through the }\end{array}$ & $\begin{array}{l}\text { Drafting error on } \\
\text { draft machine } \\
\text { when the loading }\end{array}$ & 144 & $\begin{array}{c}\text { Beginning of } \\
\text { weaving after } \\
\text { loading full yarn }\end{array}$ & $\begin{array}{l}\text { Checking of } \\
\text { original fabric } \\
\text { and fabric on }\end{array}$ & 108 \\
\hline
\end{tabular}


Yönetim ve Ekonomi Araștırmalart Dergisi / Journal of Management and Economics Research

Cilt/Volume: $16 \quad$ Sayl/Issue: 2 Haziran/June 2018 ss./pp. 162-178

M.T. Bilişik Doi: http://dx.doi.org/10.11611/yead.398967

\begin{tabular}{|c|c|c|c|c|c|c|c|c|}
\hline & & & $\begin{array}{l}\text { warp wise } \\
\text { because of } \\
\text { draft fault }\end{array}$ & $\begin{array}{c}\text { time of yarn on } \\
\text { beam }\end{array}$ & & $\begin{array}{l}\text { on beam, to } \\
\text { compare original } \\
\text { fabric and fabric } \\
\text { on the machine }\end{array}$ & $\begin{array}{c}\text { the machine are } \\
\text { provided }\end{array}$ & \\
\hline 8 & $\begin{array}{c}\text { Weaving } \\
\text { Part }\end{array}$ & $\begin{array}{l}\text { Loose } \\
\text { fabric }\end{array}$ & $\begin{array}{c}\text { Variation of } \\
\text { weft density } \\
\text { because of } \\
\text { İrregular } \\
\text { working } \\
\text { condition of } \\
\text { Warp let-off } \\
\text { motion }\end{array}$ & $\begin{array}{l}\text { Disproportion of } \\
\text { Warp let-off } \\
\text { motion }\end{array}$ & 80 & $\begin{array}{l}\text { To make periodic } \\
\text { control to Warp } \\
\text { let-off motion }\end{array}$ & $\begin{array}{l}\text { Warp let-off } \\
\text { motion peridic } \\
\text { control is done }\end{array}$ & 60 \\
\hline 9 & $\begin{array}{c}\text { Weaving } \\
\text { Part }\end{array}$ & Lattice & $\begin{array}{c}\text { Because of } \\
\text { low slashing } \\
\text { level, broken } \\
\text { warp yarn } \\
\text { directly goes } \\
\text { to weaving } \\
\text { part instead of } \\
\text { going insde of } \\
\text { drop wire }\end{array}$ & $\begin{array}{l}\text { Low slashing } \\
\text { level cause higher } \\
\text { friction which } \\
\text { cause yarn } \\
\text { breakages. }\end{array}$ & 72 & $\begin{array}{l}\text { To check every } \\
\text { slashing level } \\
\text { before loading th } \\
\text { weaving machine }\end{array}$ & $\begin{array}{l}\text { Slashing level } \\
\text { control are } \\
\text { provided for } \\
\text { evey yarn on } \\
\text { beam. }\end{array}$ & 54 \\
\hline $\begin{array}{l}1 \\
0\end{array}$ & $\begin{array}{c}\text { Weaving } \\
\text { Part }\end{array}$ & $\begin{array}{c}\text { Yarn in } \\
\text { Shed }\end{array}$ & $\begin{array}{l}\text { Small weft } \\
\text { yarn in shed }\end{array}$ & $\begin{array}{c}\text { Weft yarn knife } \\
\text { dull or abnormal } \\
\text { shed closere } \\
\text { degree. }\end{array}$ & 96 & $\begin{array}{c}\text { To make more } \\
\text { periodical for } \\
\text { Weft yarn knife } \\
\text { and shed closere } \\
\text { degree. }\end{array}$ & $\begin{array}{c}\text { Periodical } \\
\text { control of Weft } \\
\text { yarn knife and } \\
\text { shed closere } \\
\text { degree are } \\
\text { provided }\end{array}$ & 48 \\
\hline
\end{tabular}

At below table, fault possibilities, density values and detectability values of FMEA study for the relevant product were calculated . 
Table 4. Weaving Part First and Final RPN Comparison

\begin{tabular}{|c|c|c|c|c|c|c|c|c|c|}
\hline $\begin{array}{c}\text { Function } \\
\text { Mode } \\
\text { Line }\end{array}$ & Severity & Occurrence & Detection & $\begin{array}{l}\text { First } \\
\text { RPN } \\
\text { Value }\end{array}$ & Severity & Occurrence & Detection & $\begin{array}{l}\text { Final } \\
\text { RPN } \\
\text { Value }\end{array}$ & $\begin{array}{c}\text { RPN } \\
\% \\
\text { Change }\end{array}$ \\
\hline 1 & 6 & 6 & 5 & 180 & 6 & 4 & 5 & 120 & 33 \\
\hline 2 & 9 & 3 & 4 & 108 & 9 & 2 & 4 & 72 & 33 \\
\hline 3 & 5 & 4 & 5 & 100 & 5 & 3 & 5 & 75 & 25 \\
\hline 4 & 7 & 4 & 4 & 112 & 7 & 2 & 4 & 56 & 50 \\
\hline 5 & 6 & 6 & 6 & 216 & 6 & 3 & 6 & 108 & 50 \\
\hline 6 & 7 & 7 & 3 & 147 & 7 & 5 & 3 & 105 & 29 \\
\hline 7 & 6 & 4 & 6 & 144 & 6 & 3 & 6 & 108 & 25 \\
\hline 8 & 4 & 4 & 5 & 80 & 4 & 3 & 5 & 60 & 25 \\
\hline 9 & 3 & 4 & 6 & 72 & 3 & 3 & 6 & 54 & 25 \\
\hline 10 & 4 & 4 & 6 & 96 & 4 & 2 & 6 & 48 & 50 \\
\hline
\end{tabular}

As we investigate causes of faults and undertaken activities, undesirable machine aparatus works were seen because of insufficient maintenance. We focused on increasing mechanical control of machines and educations of maintenance team. So we reached the $34,5 \%$ success with undertaken activities that we have done.

\subsection{Dyeing and Finishing Part}

Dyehouse is the last department of production. In that plant, they apply both bobbin dying and fabric dying. Fabrics are dyed and colored based on customer request and send out after quality control. So, to get high quality goods as customers wish, we need the best finishing process. We found 8 available and potential error with FMEA team. 
Figure 3.

\section{Dyeing And}

\begin{tabular}{|c|c|}
\hline & Upper Bottom Twisting Error \\
\hline Upper Bottom Equipment Error \\
\hline
\end{tabular}

Error Types For

Finishing Part

Based on described error in dyehouse, as firts step, we determined effects of the failures and causes of failures with FMEA Team. Hereby, we reached First RPN Value.

After our actions with FMEA team to errors, we got Final RPV Value for each fault which showed at below table;

Table 5. Dyeing And Finishing Part Fmea Application

\begin{tabular}{|c|c|c|c|c|c|c|c|c|}
\hline & $\begin{array}{l}\text { Functio } \\
\text { n Mode }\end{array}$ & $\begin{array}{l}\text { Failure } \\
\text { Modes }\end{array}$ & $\begin{array}{l}\text { Effects of the } \\
\text { failure }\end{array}$ & $\begin{array}{l}\text { Causes of } \\
\text { Failures }\end{array}$ & $\begin{array}{c}\text { First } \\
\text { RPN } \\
\text { Valu } \\
\text { e }\end{array}$ & $\begin{array}{c}\text { Recommended } \\
\text { Activity }\end{array}$ & $\begin{array}{c}\text { Undertaken } \\
\text { Activity }\end{array}$ & $\begin{array}{c}\text { Final } \\
\text { RPN } \\
\text { Valu } \\
\text { e }\end{array}$ \\
\hline 1 & $\begin{array}{l}\text { Dyeing } \\
\text { Process }\end{array}$ & Abrage & $\begin{array}{c}\text { Stained or } \\
\text { abnormal } \\
\text { appearance on } \\
\text { surface of fabric }\end{array}$ & $\begin{array}{c}\text { Abnormal } \\
\text { dyestuff } \\
\text { dissolution or } \\
\text { machine speed or } \\
\text { undasirable } \\
\text { temperature } \\
\text { increase }\end{array}$ & 175 & $\begin{array}{l}\text { Operator education } \\
\text { regarding optimum } \\
\text { dyeing conditions }\end{array}$ & $\begin{array}{l}\text { Operators } \\
\text { have been } \\
\text { educated } \\
\text { about } \\
\text { optimum } \\
\text { dyeing } \\
\text { conditions }\end{array}$ & 140 \\
\hline 2 & $\begin{array}{l}\text { Dyeing } \\
\text { Process }\end{array}$ & $\begin{array}{l}\text { Upper } \\
\text { bottom } \\
\text { twistin } \\
\text { g error }\end{array}$ & $\begin{array}{c}\text { Uneven dyeing } \\
\text { on bobbin dying } \\
\text { process because } \\
\text { of bobbin } \\
\text { winding }\end{array}$ & $\begin{array}{l}\text { Getting not to } \\
\text { desired density } \\
\text { bobbin winding } \\
\text { because of } \\
\text { machine } \\
\text { adjustment }\end{array}$ & 100 & $\begin{array}{l}\text { Checking bobbin } \\
\text { regularly density of } \\
\text { bobbin before } \\
\text { bobbin dyeing and } \\
\text { giving information } \\
\text { to yarn production } \\
\text { part }\end{array}$ & $\begin{array}{l}\text { They checked } \\
\text { the bobbin } \\
\text { density more } \\
\text { frequent and } \\
\text { gave info to } \\
\text { production }\end{array}$ & 60 \\
\hline
\end{tabular}




\begin{tabular}{|c|c|c|c|c|c|c|c|c|}
\hline & & & & & & & side & \\
\hline 3 & $\begin{array}{l}\text { Dyeing } \\
\text { Process }\end{array}$ & $\begin{array}{c}\text { Upper } \\
\text { bottom } \\
\text { equipm } \\
\text { ent } \\
\text { error }\end{array}$ & $\begin{array}{c}\text { On bobbin } \\
\text { dyeing process, } \\
\text { inhomogeneous } \\
\text { bobbin dyeing } \\
\text { by dyestuff }\end{array}$ & $\begin{array}{l}\text { Changing dyestuff } \\
\text { quantity which is } \\
\text { passing through } \\
\text { the bobbin } \\
\text { because of } \\
\text { dyestuff leakage } \\
\text { on dyeing } \\
\text { machine }\end{array}$ & 120 & $\begin{array}{l}\text { To make periodical } \\
\text { check some points } \\
\text { which provide } \\
\text { movement of } \\
\text { dyestuff in machine }\end{array}$ & $\begin{array}{l}\text { periodical } \\
\text { check is } \\
\text { provided }\end{array}$ & 60 \\
\hline 4 & $\begin{array}{l}\text { Dyeing } \\
\text { Process }\end{array}$ & $\begin{array}{c}\text { Fastnes } \\
\text { s }\end{array}$ & $\begin{array}{l}\text { Low fastness } \\
\text { affect of dyetuff } \\
\text { after dyeing } \\
\text { process }\end{array}$ & $\begin{array}{l}\text { insufficient } \\
\text { dyeing } \\
\text { temperature, not } \\
\text { done right } \mathrm{PH} \\
\text { condition and } \\
\text { suitable chemicals }\end{array}$ & 210 & $\begin{array}{l}\text { To educate the } \\
\text { operators regarding } \\
\text { optimum dyeing } \\
\text { conditions during } \\
\text { dyeing operation }\end{array}$ & $\begin{array}{l}\text { Operators are } \\
\text { educated } \\
\text { about dying } \\
\text { prosess } \\
\text { standats. }\end{array}$ & 126 \\
\hline 5 & $\begin{array}{l}\text { Dyeing } \\
\text { Process }\end{array}$ & $\begin{array}{l}\text { Dye } \\
\text { trace }\end{array}$ & $\begin{array}{c}\text { This is a } \\
\text { unevenness } \\
\text { effect such as } \\
\text { stripre on } \\
\text { surface of fabric } \\
\text { after dyeing }\end{array}$ & $\begin{array}{l}\text { Low-speed } \\
\text { machine than } \\
\text { optimum conditon } \\
\text { or non- return of } \\
\text { fabric in machine }\end{array}$ & 147 & $\begin{array}{l}\text { To make more } \\
\text { periodical check fpr } \\
\text { some machine part } \\
\text { which provide } \\
\text { movemet to fabric }\end{array}$ & $\begin{array}{l}\text { More } \\
\text { periodical } \\
\text { check is } \\
\text { provided }\end{array}$ & 105 \\
\hline 6 & $\begin{array}{l}\text { Dyeing } \\
\text { Process }\end{array}$ & felting & $\begin{array}{l}\text { Undesirable } \\
\text { furry surface on } \\
\text { fabric after } \\
\text { dyeing process }\end{array}$ & $\begin{array}{c}\text { Higher dyeing } \\
\text { period, } \\
\text { overheating of } \\
\text { fabric or Poorness } \\
\text { of some } \\
\text { chemicals }\end{array}$ & 144 & $\begin{array}{l}\text { To educate the } \\
\text { operators regarding } \\
\text { optimum dyeing } \\
\text { conditions during } \\
\text { dyeing operation }\end{array}$ & $\begin{array}{l}\text { Operators are } \\
\text { educated } \\
\text { about dying } \\
\text { prosess } \\
\text { standats. }\end{array}$ & 96 \\
\hline 7 & $\begin{array}{l}\text { Dyeing } \\
\text { Process }\end{array}$ & $\begin{array}{l}\text { dye } \\
\text { stain }\end{array}$ & $\begin{array}{l}\text { Stains on fabric } \\
\text { because of low } \\
\text { dissolution } \\
\text { effect of some } \\
\text { dyestuff }\end{array}$ & $\begin{array}{l}\text { Poor cleaning of } \\
\text { dyeing machine, } \\
\text { Low dyestuff } \\
\text { affinity to the } \\
\text { fabric }\end{array}$ & 125 & $\begin{array}{l}\text { To educate the } \\
\text { operators regarding } \\
\text { optimum machine } \\
\text { cleaning and } \\
\text { chosing suitable }\end{array}$ & $\begin{array}{c}\text { optimum } \\
\text { machine } \\
\text { cleaning and } \\
\text { chosing } \\
\text { suitable }\end{array}$ & 75 \\
\hline
\end{tabular}




\begin{tabular}{|c|c|c|c|c|c|c|c|c|}
\hline & & & & & & dyestuff & $\begin{array}{l}\text { dyestuff are } \\
\text { provided }\end{array}$ & \\
\hline 8 & $\begin{array}{l}\text { Dyeing } \\
\text { Process }\end{array}$ & $\begin{array}{l}\text { uncolo } \\
\text { ured } \\
\text { yarn }\end{array}$ & $\begin{array}{l}\text { Stripe issue on } \\
\text { fabric because } \\
\text { of undyed warp } \\
\text { yarn }\end{array}$ & $\begin{array}{l}\text { circulating pumps } \\
\text { are on the blink }\end{array}$ & 100 & $\begin{array}{l}\text { To make more } \\
\text { periodical check } \\
\text { to circulating } \\
\text { pumps }\end{array}$ & $\begin{array}{l}\text { Run properly } \\
\text { of circulating } \\
\text { pumps are } \\
\text { provided }\end{array}$ & 40 \\
\hline
\end{tabular}

At below table, fault possibilities, density values and detectability values of FMEA study for the relevant product were calculated.

Table 6. Dyeing and Finishing Part First and Final RPN Comparison

\begin{tabular}{|c|c|c|c|c|c|c|c|c|c|}
\hline $\begin{array}{c}\text { Function } \\
\text { Line }\end{array}$ & $\begin{array}{c}\text { Severit } \\
\mathbf{y}\end{array}$ & $\begin{array}{c}\text { Occurrenc } \\
\mathbf{e}\end{array}$ & $\begin{array}{c}\text { Detectio } \\
\mathbf{n}\end{array}$ & $\begin{array}{c}\text { First } \\
\text { RPN }\end{array}$ & $\begin{array}{c}\text { Severit } \\
\mathbf{y}\end{array}$ & $\begin{array}{c}\text { Occurrenc } \\
\mathbf{e}\end{array}$ & $\begin{array}{c}\text { Detectio } \\
\mathbf{n}\end{array}$ & $\begin{array}{c}\text { Final } \\
\text { RPN }\end{array}$ & $\begin{array}{c}\text { RPN \% } \\
\text { Change } \\
\text { Value }\end{array}$ \\
\hline 1 & 7 & 5 & 5 & 175 & 7 & 4 & 5 & 140 & 20 \\
\hline 2 & 5 & 5 & 4 & 100 & 5 & 3 & 4 & 60 & 40 \\
\hline 3 & 5 & 6 & 4 & 120 & 5 & 3 & 4 & 60 & 50 \\
\hline 4 & 7 & 5 & 6 & 210 & 7 & 3 & 6 & 126 & 40 \\
\hline 5 & 7 & 7 & 3 & 147 & 7 & 5 & 3 & 105 & 29 \\
\hline 6 & 6 & 6 & 4 & 144 & 6 & 4 & 4 & 96 & 33 \\
\hline 7 & 5 & 5 & 5 & 125 & 5 & 3 & 5 & 75 & 40 \\
\hline 8 & 4 & 4 & 100 & 5 & 2 & 4 & 40 & 60 \\
\hline
\end{tabular}




\section{FINDINGS AND CONCLUSION}

When we investigate causes of faults and undertaken activities, lack of knowledge of workers and non standardisation proses are seen as main problem. We made a standard prosess especially for on dyeing diagram. So we have got $39 \%$ success in dyehouse.

As we check the source of problem of all departmant, we reach the insufficient working of some aparatus, careless working of operators as main cause. So, we determined periodic control to relevant machine and aparatus as 1 month and 3 month. Thereby, problems are be almost zeroized before taking place. In case of workers education, they organize meeting to self-improvement of them to get systematic approach for problem issue.

Objective for production companies is to reach the desired quality level by continuous improvement. Determining defect types of the process related with product to be produced, evaluating the future effects of the defects on the customers, decreasing even removing of the defects that may arise in production, determining and application of control for preventing activities shall provide a range of benefits.

\section{REFERENCES}

Akın, B. (1998) “ISO 9000 Uygulamasında İşletmelerde Hata Türü ve Etkileri Analizi”, Bilim Teknik Yayın Evi, İstanbul.

Anleitner, A. M. (2010) “The Power of Deduction Failure Modes and Effects Analysis for Design", American Society for Quality, Quality Press, Milwaukee.

Bertsche, B.,(2008) "Rehability in Automotiv and Mechanical Engineering", Springer-Verlag, Berlin Heidelberg, France.

Braaksmaa, A.J.J., Klingenberg, W., Veldman, J.(2012) “A Quantitative Method for Failure Mode and Effects Analysis", International Journal of Production Research, 50(23): 6904-6917, 1 December 2012.

Eleren, A. (2007) "Eğitim Başarısının Artırılmasında Süreç Geliştirme Yöntemlerinin Kullanılması ve Bir Uygulama", Afyon Kocatepe Üniversitesi İ̈BF Dergisi, 9 (2): 1-25.

Muller, A.(1995) “Applying Quality Management in Manufacturing at BASF", Managing Service Quality, 5 (2): 33-37.

Özyazgan, V.(2014) "FMEA Analysis and Implementation in a Textıle Factory Producing Woven Fabric”, Journal of Textile \& Apparel / Tekstil ve Konfeksiyon, 24(3). 
Raymond, J.M., McDermott,E.R., Beauregard, E.M. (2008) "The Basics of FMEA", CRC Press, Taylor \& Francis.

Roszak, M., Spilka, M., Kania A.(2015) "Environmental Failure Mode And Effects Analysis (FMEA) - A New Approach To Methodology”, Metalurgija 54 (2): 449-451.

Sabır, E. C., Bebekli, M., 2015 "Hata Türleri ve Etkileri Analizinin, HTEA, Tekstil Boya-Terbiye İşletmelerinde Kullanımı”, Çukurova Üniversitesi Mühendislik Mimarlık Fakültesi Dergisi, 28(1): 157-162, Aralık 2015.

Stamatis, D. H., (2003) "Failure Mode and Effects Analysis: FMEA from Theory of Execution", American Society for Quality, Quality Press, Milwaukee.

Vykydal, D., Plura, J.,Halfarova, P., Klaput, P. (2015) “Advanced Approaches to Failure Mode and Effect Analysis (FMEA) Applications", Metalurgija 54 (4): 675-678.

Yakit, O.(2011) "The Comparison of The Risk Priority Number Calculation Methods That Are Used In The Failure Mode Effects Analysis: Sample of Gamateks Textile Industry and Trade Inc", Süleyman Demirel Üniversitesi Vizyoner Dergisi, 3(5): 107-123.

Yılmaz, B.S.(2009) “Hata Türü ve Etki Analizi”, Dokuz Eylül Üniversitesi Sosyal Bilimler Enstitüsü Dergisi, 2(4). 